\title{
A Novel Pair-wise Recognition Scheme for Handwritten Characters in the Framework of a Multi-expert Configuration
}

\author{
A. F. R. Rahman and M.C. Fairhurst \\ Electronic Engineering Laboratory, University of Kent, Canterbury, Kent, CT2 7NT, \\ United Kingdom.
}

\begin{abstract}
A novel pair-wise recognition scheme for the recognition of handwritten characters is presented. The recognition scherne is based on using multiple neural networks to process the handwritten characters grouped in pairs. An intelligent combination scheme to combine the decisions of these individually formed and trained neural networks is developed and an overall decision tree for the identification of separate classes is realised. The whole concept is implemented and tested in the context of the classification of handwritten numerals and a substantial performance enhancement is gained.
\end{abstract}

Indexing terms: Neural networks, decision combination, handwritten character recognition, multiple expert classifiers.

\section{Introduction}

Techniques based on the combination of decisions of multiple classifiers to enhance the overall performance of a pattern recognition system have been the focus of much research in the recent years ( [1], [2] and [3] ). It is being recognised that the use of multiple experts can often offer decisions about classifications with a greater degree of reliability and robustness. This paper describes a novel recognition scheme based on combining pairwise classification decisions of multiple neural networks and defines a decision tree to arrive at a final decision about the identity of the input patterns. The whole concept is implemented and tested in the context of the classification of handwritten numerals. The scheme relies on the proper formation of pairs among the input pattern classes and effort has been directed to the optimisation of the overall system to achieve the highest possible recognition rate with no rejection. The following sections describe the concepts involved in this scheme and also illustrate how the concepts can be implemented in the framework of a practical multiple expert configuration.

\section{Multiple Expert Classification}

The philosophy behind this multilayer classification scheme is to process pairs of symbols together. The criterion of selection of the pairs is the inherent structural 
similarity that is present in the symbols. A simple way to determine similarities among the input patterns is to use the Hamming Distances between the symbols. The idea is to separate the pairs which are likely to be confused with each other and process them separately. The proposed structure is a multiple stage structure where the initial stage consists of specialised separators trained individually to separate different sets of symbols for further processing. Negative training is applied to each of these specialised separators to reject the symbols which do not belong to that particular set. Depending on whether the separators are single structures or multiple structures, two different types of multiple expert classifiers can be realised.

- Type-I : The separators perform the dual function of separation and classification. For example, if a separator is intended to be used for the pair ' $1 / \gamma$ ', then it should perform the following functions:

- Classify ' 1 ' and ' $\gamma$ ' into separate classes.

- Reject any other numeral.

- Type-II : The separators perform the function of separation only. For example, if a separator is intended to be used for the pair ' $3 / 8$ ', then it should perform the following functions:

- Form a cluster which includes both ' 3 ' and ' 8 '.

- No separation into separate classes between ' 3 ' and ' 8 ' is performed.

- Reject any other numeral.

Figure 1 demonstrates how a multiple expert system employing Type-I separators can be used for efficient multiple-class classification. Figure 2, on the other hand, illustrates how Type-II separators are used in conjunction with other experts to achieve efficient multiple-class classification. The following is a short description of how numeral classification takes place in the framework of this multiple expert system.

\subsection{Classification Strategy for Classifiers Employing Type-I Separators}

In structures employing Type-I separators, these separators form the primary layer of the multiple layer configuration. In the specific problem of the classification of handwritten numerals, five independent separators are designed. Each of these separators corresponds to a defined pair of numerals, each separator producing two recognition responses and one rejection response. All of these separators work in parallel and each random input symbol is presented simultaneously to all of these separators. The responses produced by these separators are evaluated by a response evaluation expert coming immediately after the separators. Response evaluation is essential as all the separators produce responses corresponding to the same input symbol (a numeral character in this example). The algorithm developed in order to assess the responses is as follows: 
Random Input Symbol Stream

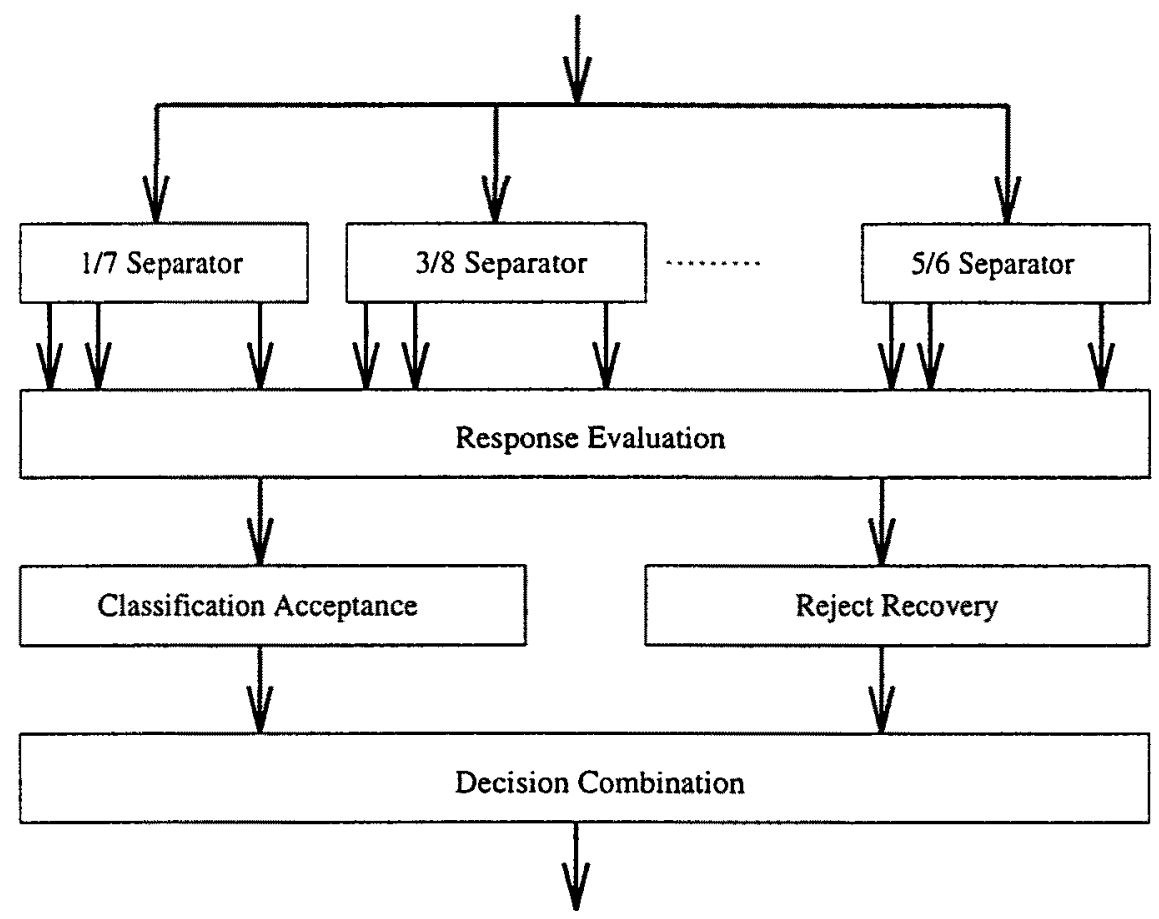

Final Decision

Fig. 1. Logical structure of a multi-expert system incorporating Type-I separators.

- 1. A response is valid only if the score produced by the neural network separators is more than a threshold value, otherwise it is ignored.

- 2. When the responses include multiple acceptance and rejection, ignore the rejection responses and follow Rule-3.

- 3. Considering all the recognition responses produced by all the separators, assign the test pattern to the class corresponding to the separator response having the highest score.

- 4. If more than one recognition response gives the same score, assign the test pattern arbitrarily to one of the classes corresponding to the separators producing that score.

- 5. If all the valid responses belong to the rejection group, then reject the test pattern.

All the rejected test patterns are re-evaluated by a reject recovery expert (see, for example, [4], [5] and [6]) coming next in the hierarchy of the multiple expert system. The reject recovery expert produces an $n_{-} b y_{-} n$ response, when there are $n$ classes under consideration and operates with no rejection. Finally, all the decisions made by the evaluators and reject recovery experts are combined and a final decision is reached. 
Random Input Symbol Stream

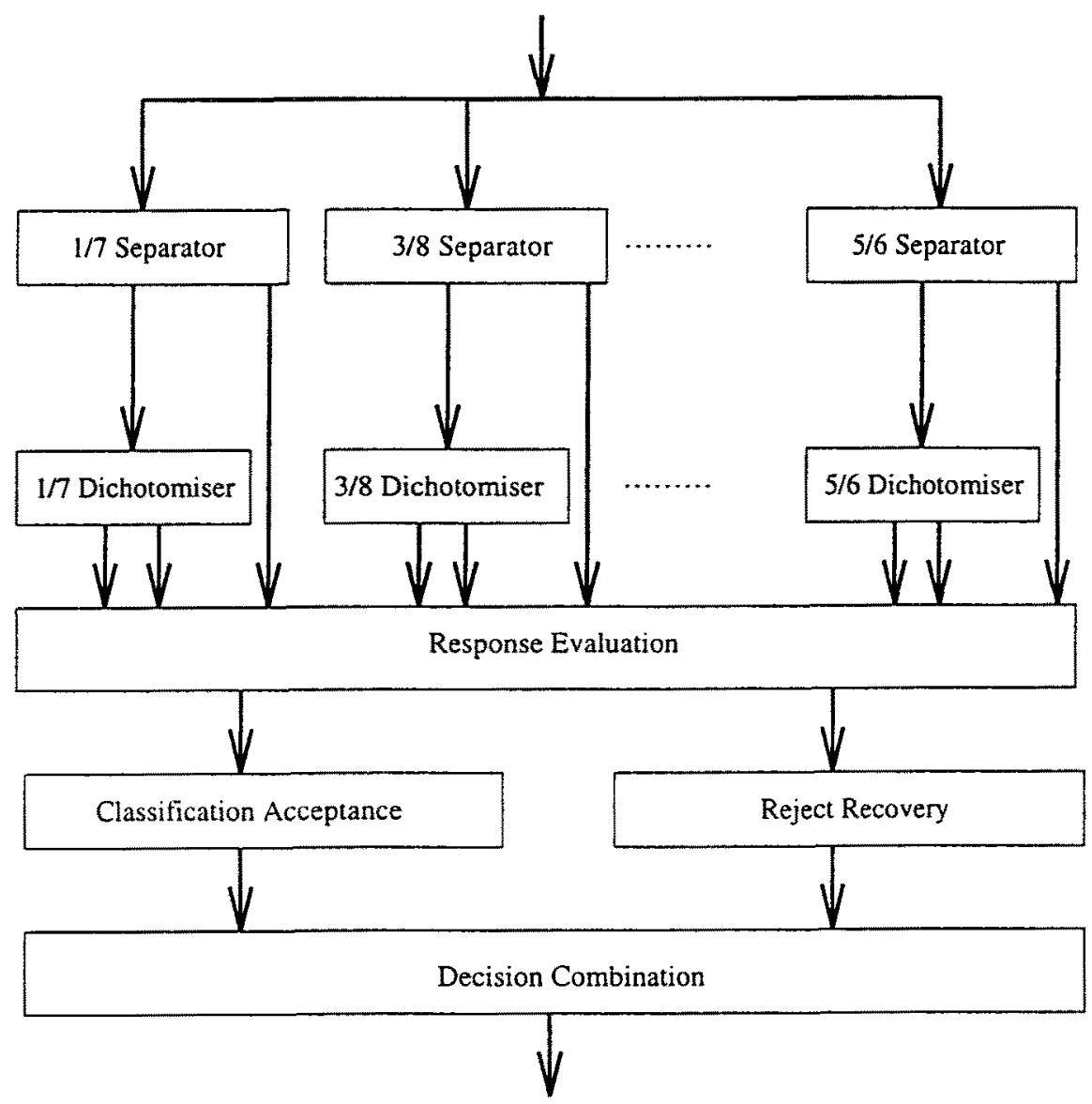

Final Decision

Fig. 2. Logical structure of a multi-expert system incorporating Type-II separators.

It can be seen that these multiple expert configurations have four levels of decision hierarchy, where of these four levels, two are active layers and two are passive layers. The layers incorporating the separators and reject recovery experts form the active layers and the other two layers form the passive layers. They are differentiated in this fashion because it is only in the active layers that computations regarding feature extraction, prototyping and classification take place. The passive layers only take decisions based on logical reasoning using information previously computed by the active layers. 


\begin{tabular}{|c|c|}
\hline \multicolumn{2}{|c|}{ Overall Recognition Performance } \\
\hline Single Expert & Multiple Experts \\
\hline$(\mathrm{NN})$ & Type-I Separator Type-II Separator \\
\hline 92.1 & 93.3 \\
\hline
\end{tabular}

Table 1. Performance comparison between single expert and multi-expert system.

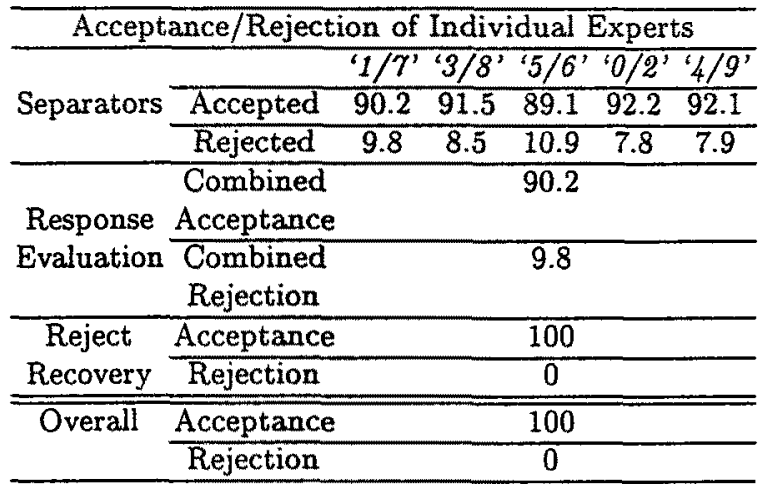

Table 2. Acceptance and rejection of individual experts (Type-I separators)

\subsection{Classification Strategy for Classifiers Employing Type-II Separators}

In structures employing Type-II separators, these separators form the primary layer of the multiple expert configuration, in just the same way as in the case of the structures that employ Type-I separators. The difference in this case is that the structure employs one more active layer to take care of the final separation of the pairs extracted by the Type-II separators. As already described in section 2, Type-II separators only perform a separation of the target pairs, but do not perform any final classification. These separated pairs themselves need to be separated into individual classes. As the classifiers employed in this layer only need to perform a two-way classification, they are dichotomisers. These dichotomisers form the second active layer of this multiple expert configuration. After individual classification has been performed by the dichotomisers, response evaluation takes place exactly in the same way as is performed in the previouslydescribed configurations based on Type-I separators.

\section{Experiments and Results}

The performance of the multi-expert system was evaluated on a reference database of handwritten pre-segmented numerals $(0,1, \ldots, 9)$. Since a multi-expert configuration is only of practical value if the final overall performance based on the combination of all the individual decisions outperform the individual performances 


\begin{tabular}{|c|c|c|c|c|c|c|}
\hline \multicolumn{7}{|c|}{ Acceptance/Rejection of Individual Experts } \\
\hline \multirow{3}{*}{ Separators } & & $\overline{' 1 / 7}$ & $3 / 8^{\prime}$ & $5 / 6$ & $0 / 2$ & $\sqrt[4 / 9^{\prime}]{4}$ \\
\hline & Accepted & 92.7 & 94.3 & 93.1 & 94.2 & $\overline{93.1}$ \\
\hline & Rejected & 7.3 & 4.7 & 6.9 & 5.8 & 6.9 \\
\hline \multirow[t]{2}{*}{ Dichotomisers } & Accepted & 100 & 100 & 100 & 100 & 100 \\
\hline & Rejected & 0 & $\overline{0}$ & 0 & 0 & $\overline{0}$ \\
\hline \multirow{3}{*}{$\begin{array}{l}\text { Response } \\
\text { Evaluation }\end{array}$} & Combined & \multirow{2}{*}{\multicolumn{5}{|c|}{93.8}} \\
\hline & Acceptance & & & & & \\
\hline & $\begin{array}{l}\text { Combined } \\
\text { Rejection }\end{array}$ & \multicolumn{5}{|c|}{6.2} \\
\hline \multirow{2}{*}{$\begin{array}{c}\text { Reject } \\
\text { Recovery }\end{array}$} & Acceptance & \multicolumn{5}{|c|}{100} \\
\hline & Rejection & \multicolumn{5}{|c|}{0} \\
\hline \multirow[t]{2}{*}{ Overall } & Acceptance & \multicolumn{5}{|c|}{100} \\
\hline & Rejection & \multicolumn{5}{|c|}{0} \\
\hline
\end{tabular}

Table 3. Acceptance and rejection of individual experts (Type-II separators)

of the participating experts, the proposed multi-expert system was compared with the individual experts and the overall results are shown in Table 1. This shows that the multi-expert system outperforms the individual experts in terms of overall performance, thereby satisfying the principal criterion of practical viability. An individual neural network was trained to recognise the ten classes under investigation. A more detailed study of the performances, however, reveals some interesting properties of the multi-expert system. The system depends on efficient separation of target pairs. Table 2 illustrates how, in the case when Type-I separators were used, the different components of the multiple expert system realised efficient rejection in order to re-process the target pairs. Table 3 illustrates the same rejection capacity of the system, component by component, in the case when Type-II separators are used. Table 4 shows how the different experts in the multi-expert system perform individually in the case of configurations employing Type-I separators and Table 5 shows how the equivalent experts perform in the case of configurations using Type-II separators. All the experts used in conjunction with these multiple expert configurations were Multilayer Perceptrons[7] with one hidden layer. The image resolution was $16 \times 24$. All the neural networks (NNs) had an input layer of 384 nodes and a hidden layer of 80 nodes. Depending on the type of expert, the NNs had a different number of output nodes. The individual expert which was used to compare the performance enhancement of the multiple expert had 10 output nodes corresponding to the ten numeral classes. Type-I separators had three output nodes each, while TypeII separators had two output nodes. The dichotomisers had two output nodes, but the reject recovery expert obviously had ten output nodes corresponding to the ten input classes associated with the ten handwritten numeral symbols. 


\begin{tabular}{llllll}
\hline \multicolumn{4}{c}{ Performance of Individual Experts } \\
\hline \multirow{3}{*}{ Separators $1 / 7^{\prime}{ }^{\prime} 3 / 8^{\prime}{ }^{\prime} 5 / 6^{\prime}{ }^{\prime} 0 / 2^{\prime}$} & $4 / 9^{\prime}$ \\
\cline { 2 - 5 } & Recognition 96.1 & 97.2 & 95.8 & 94.1 & 95.2 \\
\cline { 2 - 5 } & Rejection 65.2 & 69.1 & 68.8 & 72.5 & 71.3 \\
\hline Recognition & Recognition & \multicolumn{3}{c}{94.3} & \\
\cline { 2 - 5 } Performance & Rejection & 68.4 & \\
\hline $\begin{array}{c}\text { Reject } \\
\text { Recovery }\end{array}$ & Recognition & 91.5 & \\
\hline Overall Recognition & & & \\
\hline
\end{tabular}

Table 4. Overall performance of individual experts (Type-I separators)

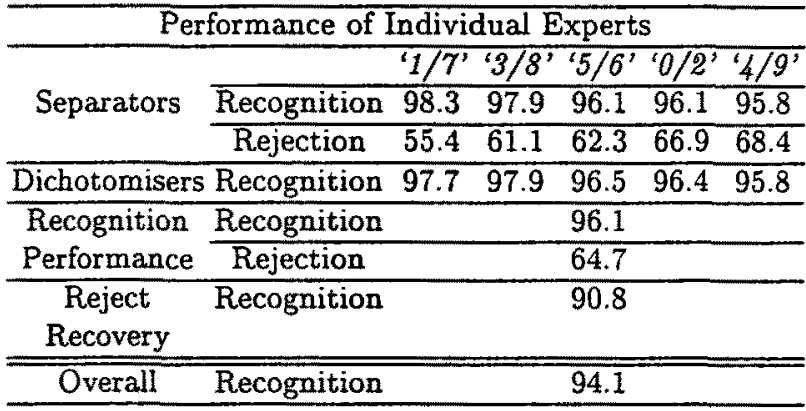

Table 5. Overall performance of individual experts (Type-II separators)

\section{Discussion of the Performance of the System}

The proposed approach to the task of handwritten numeral recognition has produced very encouraging results. It is found that efficient separation of numerals into pairs having a high degree of structural similarity helps in gaining valuable advantage in terms of more reliable pairwise classification. The configurations employing Type-I separators showed that these are less effective in recognising the target classes in comparison to the Type-II separators where the separators perform only the function of separation. The figures in Table 4 and Table 5 with respect to the rejection performance denote the real percentage of classes included in the rejected numerals. It should also be emphasised, however, that this is not particularly important, since the reject recovery expert is capable of re-classifying these discarded numerals at no rejection with a high level of accuracy. On the other hand, greater advantage can obviously be gained from using specialised dichotomisers which do perform at a very high recognition rate.

\section{Conclusions}

A novel pairwise classification scheme based on using multiple neural network classifiers has been presented and implementation details about a practical con- 
figuration for the recognition of handwritten numerals has been described. The scheme depends on efficient separation of structurally similar symbols into separate streams and then applying a specialised recognition process dedicated to specific target groups. The approach is relatively simple in concept and consideration of the structural details suggest that the system is a very good candidate for implementing in parallel software. Use of the MLP structure is obviously not a requirement in realising the experts in different layers, and any other statistical or syntactic classification methods can be used in designing the different experts. In the case of designing the separators, both Type-I and Type-II, MLPs are obvious logical choices because of their capability for negative training and efficiency in providing reliable rejection. Further research is continuing to optimise the system with respect to the efficient formation of pairs and development of other neural network structures to be used as effective separator modules in the context of this type of multiple expert configuration.

\section{References}

1. Kittler, J. and Hatel, M.: Improving recognition rates by classifier combination. Fifth International Workshop on Frontiers of Handwriting Recognition, Sep. 2-5, University of Essex, UK., (1996) 81-102.

2. Cao, J., Ahmadi, M. and Shridhar, M.: Recognition of handwritten numerals with multiple features and multistage classifier. Pattern Recognition. 28(2) (1995) 153160.

3. Waterhouse, S. R. and Robinson, A. J.: Classification using hierarchical mixtures of experts. Proc. IEEE Workshop on Neural Networks for Signal Processing IV. (1994) $177-186$.

4. Rahman, A. F. R. and Fairhurst, M. C.: A new approach to handwritten character recognition using multiple experts. Fifth International Workshop on Frontiers of Handwriting Recognition, Sep. 2-5, University of Essex, UK., (1996) 283-286.

5. Rahman, A. F. R. and Fairhurst, M. C.: Recognition of handwritten characters with a multi-expert system. IEE Workshop on Handwriting Analysis and Recognition A European Perspective, May 23, London, (1996).

6. Fairhurst, M. C. and Rahman, A. F. R.: A Generalised approach to the recognition of structurally similar handwritten characters. IEE Proc. on Vision, Image and Signal Processing, 144(1) (1997) 15-22.

7. Rumelhart, D. E., Hinton, G. E. and Williams, R. J.: Learning internal representations by error propagation. in Parallel Distributed Processing 1, Rumelhart, D. E. and McClelland, J. L., eds., MIT Press, Cambridge, MA, (1986) 318-362. 